\title{
Correction to: Semi-Supervised Sentiment Analysis of Portuguese Tweets with Random Walk in Feature Sample Networks
}

\author{
Pedro Gengo and Filipe A. N. Verri
}

\begin{abstract}
Correction to:
Chapter "Semi-cupervised Sentiment Analysis of Portuguese Tweets with Random Walk in Feature Sample Networks" in: R. Cerri and R. C. Prati (Eds.): Intelligent Systems, LNAI 12319, https://doi.org/10.1007/978-3-030-61377-8_42
\end{abstract}

Inadvertently the authors of this chapter released it without correcting an error in the title. This has now been corrected and the corrected title reads: "Semi-Supervised Sentiment Analysis of Portuguese Tweets with Random Walk in Feature Sample Networks". 